\title{
Design and Simulation of an IF MEMS Filter Based on Face Shear Mode Square Resonator
}

\author{
Junwen Jiang, Jingfu Bao*, Yijia Du \\ School of Electronic Engineering, University of Electronic Science and Technology of China, Chengdu 611731, China
}

\begin{abstract}
Intermediate frequency (IF) Filters with IC (Integrated circuit) compatibility, narrow bandwidth and large stop band rejection are in need for wireless communications. This paper presents the design and simulation of a $70 \mathrm{MHz} \mathrm{IF}$ Micro-electro-mechanical filter using two identical face shear mode square resonators. By carefully designing the supporting structure of the resonators, a quality factor above 100,000 is achieved in the best designed resonator. With these face shear mode square resonators, three filters are designed and the simulated transmission response shows that these filters can obtain unterminated transmission passbands of about $25 \mathrm{kHz}$ to $35 \mathrm{kHz}$ with the centre frequency of $70 \mathrm{MHz}$, which represents a $0.036 \%$ to $0.05 \%$ bandwidth. This ultra narrow bandwidth IF bandpass filter can be an attractive candidate for wireless receivers.
\end{abstract}

Keywords: Micro-electro-mechanical system, intermediate frequency filters, square resonator

\section{Introduction}

With the advantage of low power consumption, exceptionally high quality factor, and Integrated circuit (IC) compatibility, MEMS (Micro-electro-mechanical system) resonator and filters have become attractive as frequency generation and selection components for wireless communication systems. Typically for front end transceiver, there exist a number of high- $Q$ off-chip components that limit the development of system on chip (SOC), however, these components are replaceable by MEMS structures and devices. MEMS resonators can be served to construct MEMS oscillators as well as high-Q ultra narrow bandwidth filters, which are attractive in wireless front end receiver to achieve better signal selection. Over the past decade, many efforts had been done to the research and fabrication of MEMS filters. Frank D. Bannon used electrostatically actuated MEMS beam resonator to construct an $8 \mathrm{MHz}$ filter with bandwidth from $0.23 \%$ to $2.5 \%$ in 2000 [1]. S. Wang designed a ultra-narrow bandwidth ring filter using a balanced design, which achieved a bandwidth of $0.029 \%$ in $5.9 \mathrm{kHz}$ with $2.7 \mathrm{~dB}$ insertion loss in 2011 [2]. A bandpass filter with center frequency of $173 \mathrm{MHz}$ and bandwidth of $110 \mathrm{kHz}$ is realized with poly-SiC Lame mode resonator by Sunil A. Bhave in 2005 [3]. All these efforts are aiming at achieving lower motional impedance and narrow bandwidth for better applications.

In this paper, a face shear mode square resonator is employed for realizing ultra narrow bandwidth IF MEMS filter. Firstly, the design and operation of the unconventional mode of resonator is introduced. What's more, three different supporting structures are designed to find a better way to achieve high quality factor in this resonator. At last, a finite element modeling (FEM) simulation with ANSYS is implemented to obtain the transmission response of the filter. The results show the unterminated response with a passband about $25 \mathrm{kHz}$ to $35 \mathrm{kHz}$ in $70 \mathrm{MHz}$ centre frequency, which represents a $0.36 \%$ to $0.05 \%$ bandwidth.

\footnotetext{
* Manuscript received July 16, 2012; revised August 25, 2012.

Corresponding author. Tel.: +86-28-61830365; E-mail address: baojingfu@uestc.edu.cn
} 


\section{Structure and Operation}

The IF MEMS filter consists of two identical square resonators, mechanically coupled by a flexural mode beam as shown in Fig. 1. Two groups each with four separated electrodes are positioned on the side of the resonators, one group is for input signals and the other is for sensing.

Fig. 1. The structure of the IF MEMS filter.

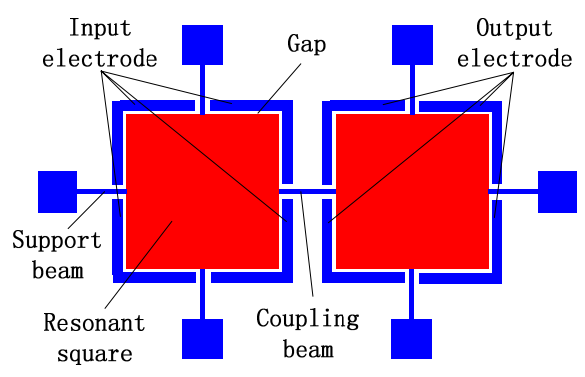

\subsection{Square resonator design}

A square resonator operating in its face shear mode is employed, whose frequency can be given by [4].

$$
f_{0}=\frac{\kappa}{L} \sqrt{\frac{G}{\rho}}
$$

where $L$ is the side length of the resonant square, $\kappa$ is the mode factor, $\rho$ is the density of the structure material and the shear modulus $G$ can be expressed as

$$
G=\frac{E}{2(1+v)}
$$

where $E$ is Young's modulus and $v$ is Poisson's coefficient. The circuit equivalent model of the resonator is depicted in Fig. 2. By using an F-V analogy the parameters of the equivalent circuit model can be represented as [1]

$$
L_{x}=\frac{m_{e q}}{\eta^{2}}, R_{x}=\frac{\sqrt{k_{e q} m_{e q}}}{Q \eta^{2}}=\frac{c_{e q}}{\eta^{2}}, C_{x}=\frac{\eta^{2}}{k_{e q}}, C_{0}=\frac{\varepsilon_{0} h W_{e}}{d_{0}}
$$

where $Q$ is the quality factor of the resonator, $h$ is the thickness of the resonance square, $W_{e}$ is the width of the input/output electrode and the transduction ratio $\eta$ can be calculated with (4), where $V_{p}$ is the DC bias voltage applied to the resonance square.

$$
\eta=V_{p} \frac{\partial C_{0}}{\partial d}
$$

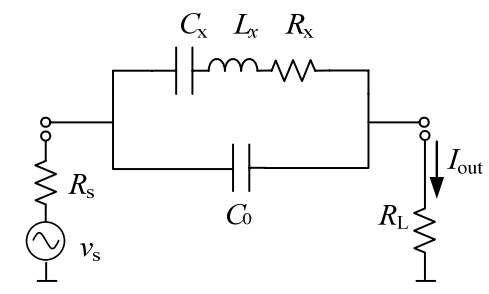

Fig. 2. Circuit equivalent model of the MEMS resonator.

When operating in vacuum the quality factor of the face shear mode square resonator is limited by the anchor loss, which is the energy dissipated though the supporting structure to the silicon base. Three different support structures are designed and simulated for the resonator: straight beam support, T-shaped 
support and cross-shaped support as shown in Fig. 3. With FEM simulation, higher $Q$ is obtained with Tshaped support and cross-shaped support than the straight beam support, which will be discussed later.

Motional impedance is also a very important factor in the application of the MEMS resonators and filters. Two basic ways to decrease the motional impedance are reducing the electrode to resonator gap and increasing the efficient electrode to resonator relative area. With recent high aspect-ratio SOI technology, a $0.5 \mu \mathrm{m}$ gap can be realized with $20 \mu \mathrm{m}$ thick of the resonant body, which could provide smaller motional impedance in lateral vibration mode MEMS resonator [5].

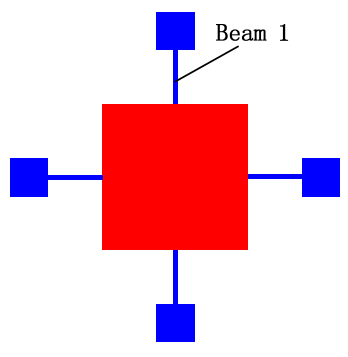

(a)

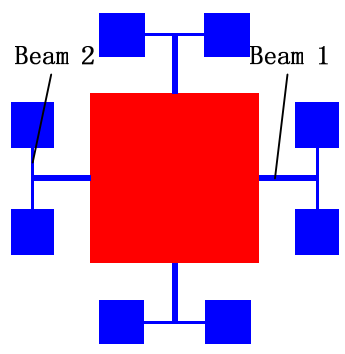

(b)

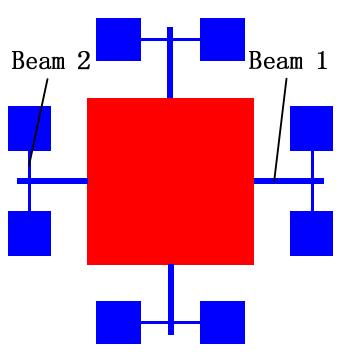

(c)

Fig. 3. Square resonator supporting structures (a) straight beam support; (b) T-shaped support; (c) cross-shaped support

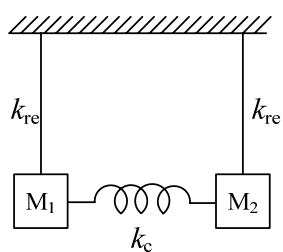

(a)

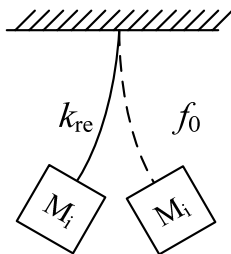

(b)

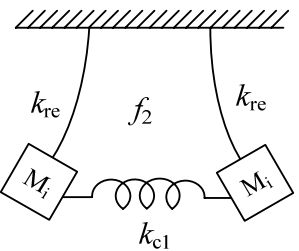

(c)

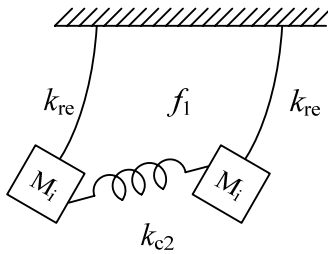

(d)

Fig. 4. Coupled spring-mass resonators (a) Resonators at rest; (b)Single resonator natural mode; (c)Out-of-phase mode; (d)In-phase mode[8].

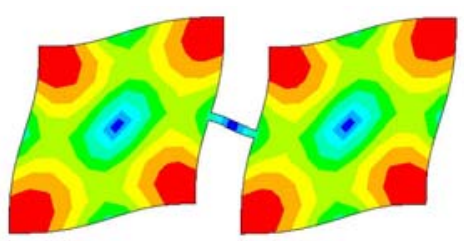

(a)

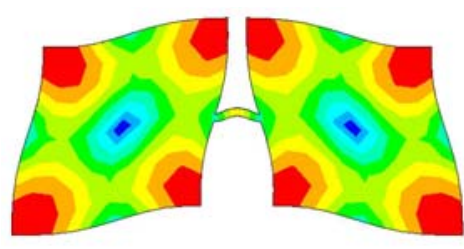

(b)

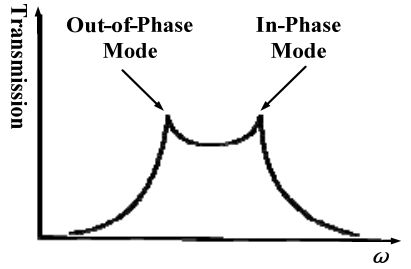

(c)

Fig. 5. Coupled resonator vibration modes (a) In-phase mode; (b) Out-of-phase mode; (c) transmission response of the filter

\subsection{MEMS filter operation}

This two-resonator-based MEMS filter has two mechanical-resonance modes with closely spaced frequencies that form the filter passband. Fig. 4 shows the equivalent lumped-mass mechanical system of the filter, and the contour profile of this filter is depicted in Fig. 5. The frequency of the constituent resonator determines the center frequency of the filter and the passband of the filter are up to the stiffness of the coupling beam and equivalent stiffness of the resonator in the coupling point. Actually, the frequency of each resonance peak can be calculated by [6]

$$
f_{1} \approx f_{0}\left(\frac{k_{r e}-k_{c 1}}{k_{r e}}\right), f_{2} \approx f_{0}\left(\frac{k_{r e}+k_{c 2}}{k_{r e}}\right)
$$

where $k_{r e}$ is the equivalent stiffness of the resonator in the coupling point, $k_{c 1}$ and $k_{c 2}$ are the stiffness of the coupling beam at out-of-face mode and in-phase mode, respectively. Consequently, the pass band can be calculated by 


$$
B=f_{2}-f_{1}=f_{0} \frac{k_{c 2}+k_{c 1}}{k_{r e}}
$$

Thus, a desired passband can be obtained by either changing the coupling point or carefully designing the dimention of the coupling beam. Also, the choice of the coupling point is significant in the output signal of the filter, precisely the vibration amplitude of the sensing resonator, which determines the output current of the filter. For better coupling in the most researched lame mode of the square resonator, either directly coupled at the corner [3] or coupled in the centre of the side separating the electrode similar as Yang Lin did in disk coupling [7]. The former method may be not a strong coupling since the corner in a lame mode is a zero vibration point while the second one reduces the output magnitude, however, the face shear mode did not face such problems when designing the coupling beam, the coupling beam can be set at the centre of the side without breaking the electrode and still be well coupled.

\section{Simulation and Results}

The parameters of the filter to be simulated are listed in Table 1.

Table 1. Parameters of the designed filter

\begin{tabular}{lc}
\hline Parameters & Values $(\mu \mathrm{m})$ \\
\hline Square side length, $L$ & 49 \\
Thickness of the square resonator, $h$ & 10 \\
Electrode to resonator gap, $g_{0}$ & 0.1 \\
Length of the support beam1, $l_{1}$ & 10 \\
Width of the support beam1, $W_{1}$ & 2 \\
Length of the support beam2, $l_{2}$ & 8 \\
Width of the support beam2, $W_{2}$ & 2 \\
Length of the coupling beams, $l_{c}$ & 6 \\
Width of the coupling beams, $W_{c}$ & 2 \\
\hline
\end{tabular}

Table 2. Parameters of the designed filter

\begin{tabular}{ll}
\hline Parameters & Values \\
\hline Quality factor with straight support & 6,500 \\
Quality factor with T-shaped support & 44,700 \\
Quality factor with cross-shaped support & 11,300 \\
\hline
\end{tabular}

A FEM simulation with ANSYS is used to get the transmission response of the designed filter. Firstly, the quality factor of the shear mode square resonator with each of the three supporting structures is simulated using an acoustic absorbing layer. The results are listed in Table 2, which show a significant improvement of $Q$ in T-shaped support and cross-shaped support comparing to straight support. Since a higher $Q$ will help to achieve a lower motional impedance as well as better rectangle coefficient of MEMS filter, a T-shaped support or cross-shaped support is strongly recommended when designing a face shear (very likely other lateral vibration mode) mode square resonator.

We use a harmonic simulation with multiphysics in ANSYS, which could combine electronic field and mechanical field in the harmonic simulation. Fig. 6 shows the FEM model and the counter plot of the result of the harmonic simulation. The resonator in Fig. 6 (a) is the actuating one for sensing input signals and the one in Fig. 6 (b) is for output signals. From Fig. 6 (b) we can see that the displacement of the output resonator is almost the same as the input resonator, this indicates a good coupling, which will help to realize higher signal to noise ratio. While in some poor coupled MEMS filters, the displacement of the output resonator is much smaller than the input resonator.

The unterminated transmissions of the MEMS filter with the three different supporting structures are depicted in Fig. 7. The centre frequency is about $70 \mathrm{MHz}$ as designed and the passband is about $2.5 \mathrm{kHz}$, $3.5 \mathrm{kHz}$ and $3.2 \mathrm{kHz}$ from (a), (b) and (c), respectively. And it can be find that the stopband is much nearer to the centre in (b) and (c) than that in (a), which indicates a better rectangular coefficient for the filter. It seems that these transmission curves do not look like filter amplitude to frequency responses, 
especially in (b) and (c), actually after a termination with right resistance, the sunk part in the curves would be almost illuminated [6].

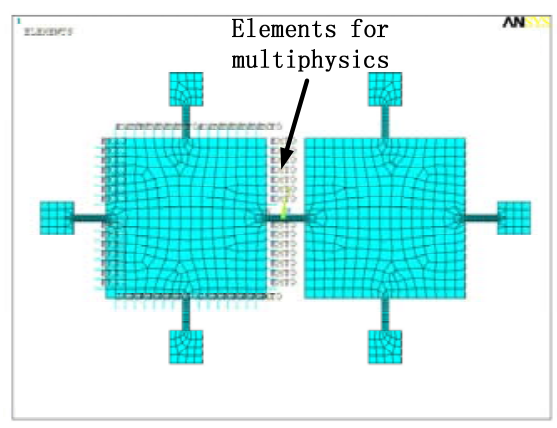

(a)

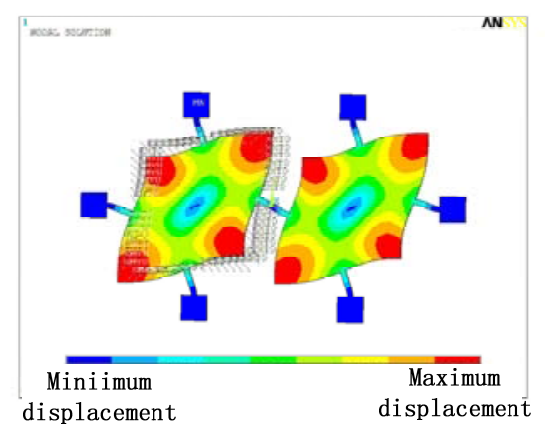

(b)

Fig. 6. Designed filter (a) FEM model for the filter, (b) harmonic simulation result

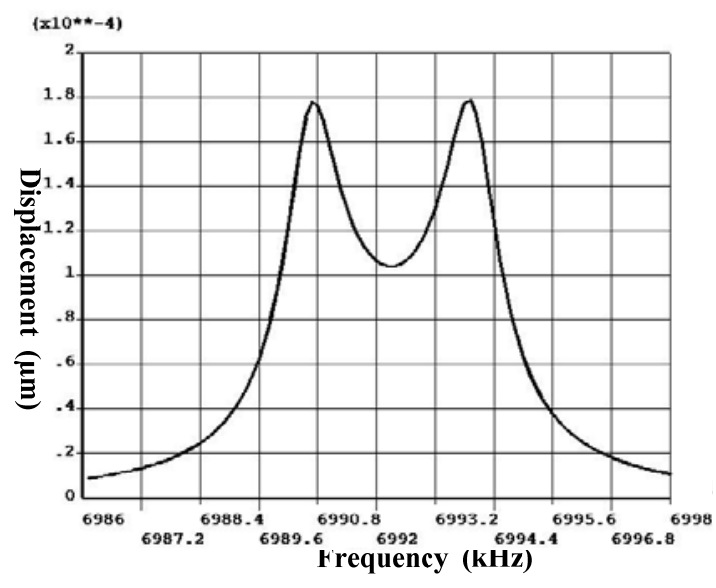

(a)

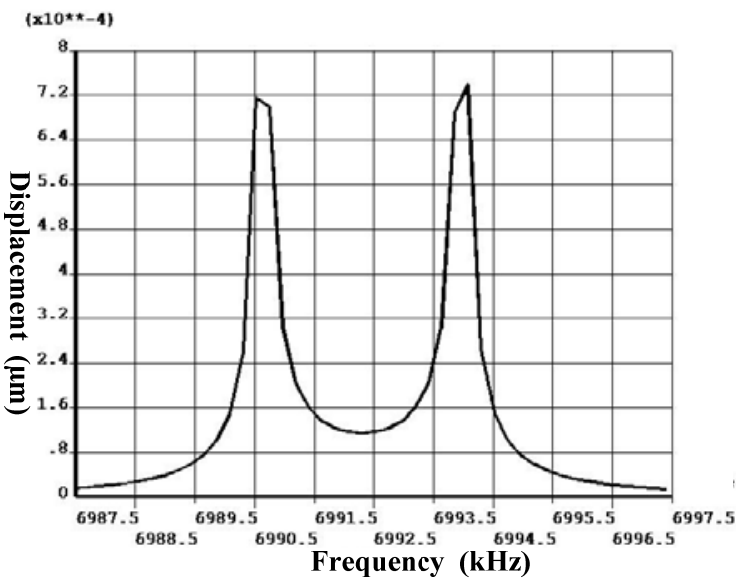

(b)

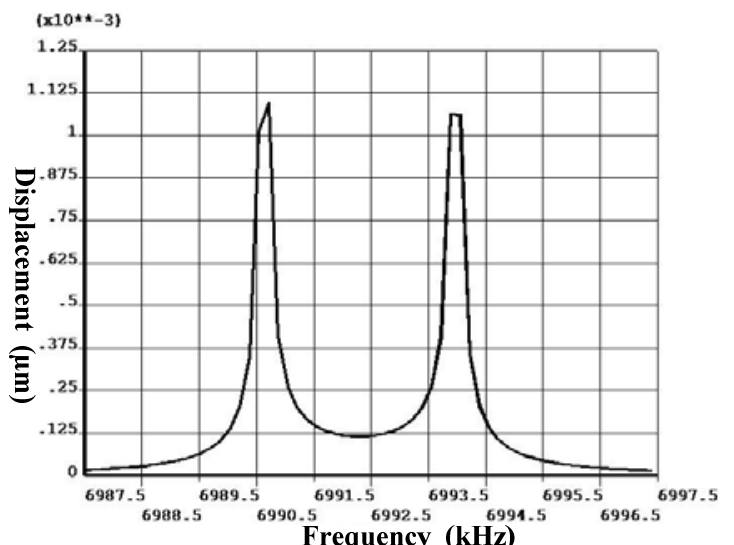

(c)

Fig. 7. Unterminated transmission of the filter: (a) with straight beam support; (b) with T-shaped support; and (c) with cross-shaped support.

\section{Conclusion}

A novel face shear mode square resonator based IF MEMS filter is designed and simulated in this paper for higher integration density transceiver in the future. The quality factor of the resonators for constructing filters is always the higher the better, consequently, we researched three different supporting structures for reducing anchor loss. The simulated results shows that the resonators with cross-shaped support and the T-shaped support obtains much high $Q$ than the straight beam supported one. With a 
coupling beam of $6 \mu \mathrm{m}$ long and $2 \mu \mathrm{m}$ width, the two resonator system constructed a MEMS filter with $70 \mathrm{MHz}$ centre frequency and a bandwidth of about 0.036 to $0.05 \%$, which is very small in bandwidth filters. The improvement of stop band rejection in the filters with T-shaped support and cross-shaped support comparing to the straight beam support is also indicated in the transmission curves.

\section{Acknowledgements}

This work was supported by the National Natural Science Foundation of China and the China Academy of Engineering Physics (11176006), the Foundation of China (Grant No. 9140A23060409DZ02)

\section{References}

[1] Bannon FD, Clark JR, Nguyen CTC. High-Q HF microelectromechanical filters. IEEE Journal of Solid-State Circuits, 2000; 35(4):512-526.

[2] Wang S, Chandorkar SA, Graham AB, Messana MW, Salvia J, Kenny TW. Encapsulated mechanically coupled fullydifferential breathe-mode ring filters with ultra-narrow bandwidth. In: Proc. of the 16th International Conference on SolidState Sensors, Actuators and Microsystems (TRANSDUCERS), 2011:942-945.

[3] Sunil A. Gao B, Maboudian R, Howe RT. Fully-differential poly-SiC Lame mode resonator and checkerboard filter. In: Proc. of IEEE International Conference on Micro Electro Mechanical Systems, 2005:223-226.

[4] Khine L, Palaniapan M. High-Q bulk-mode SOI square resonators with straight-beam anchors. J. Micromech. Microeng, 2009; 19(1):015017.

[5] Abdolvand R, Farrokh A. An advanced reactive ion etching process for very high aspect-ratio sub-micron wide trenches in silicon. Sensors and Actuators A-Physical, 2008; 144(1):109-116.

[6] Babazadeh F, Keshmiri SH. Modeling of a novel high-Q, highly linear, IF micromechanical filter: design and simulations. World Applied Sciences Journal, 2009; 6(7): 914-925.

[7] Lin Y, Li WC, Kim BS, Lin YW, Ren ZY, Nguyen CTC. Enhancement of micromechanical resonator manufacturing precision via mechanically-coupled arraying. In: Proc. of IEEE International Frequency Control Symposium, 2009:58-63.

[8] R. Johnson. Mechanical Filters in Electronics. New York: Wiley; 1987. 